\title{
L'expérience de femmes ayant subi une grossesse non désirée au Sénégal : Une étude qualitative
}

\author{
Fatou Mbow \\ Population Council \\ Nafissatou Diop \\ Population Council \\ Awa Diop Dabo \\ Awa Tounkara Cissé \\ Seynabou Ba Diakhaté
}

Follow this and additional works at: https://knowledgecommons.popcouncil.org/departments_sbsr-rh

Part of the Demography, Population, and Ecology Commons, Family, Life Course, and Society Commons, Gender and Sexuality Commons, International Public Health Commons, Maternal and Child Health Commons, and the Women's Health Commons How does access to this work benefit you? Let us know!

\section{Recommended Citation}

Mbow, Fatou, Nafissatou Diop, Awa Diop Dabo, Awa Tounkara Cissé, and Seynabou Ba Diakhaté. 2016. "L'expérience de femmes ayant subi une grossesse non désirée au Sénégal : Une étude qualitative," STEP UP Rapport de recherche. Dakar: Population Council. 


\section{STEP \\ STRENGTHENING EVIDENCE FOR PROGRAMMING ON UNINTENDED}

SENEGAL

Rapport de recherche

Juin 2016

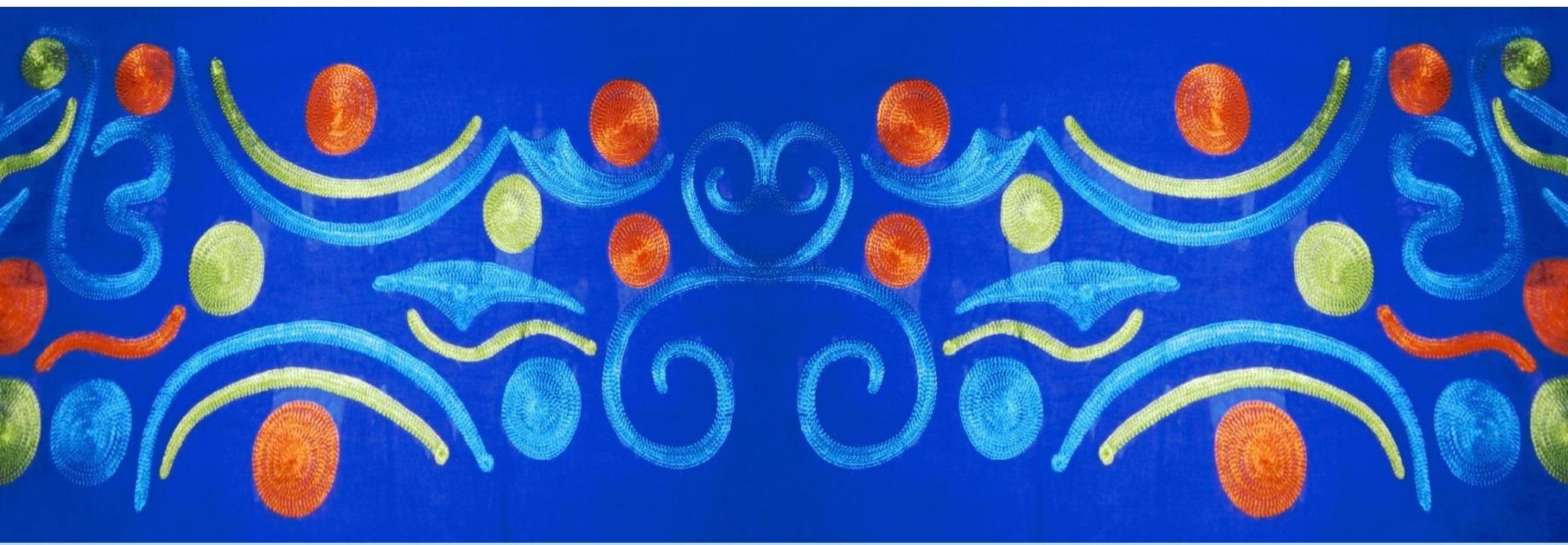

\section{L'expérience de femmes ayant subi une grossesse non désirée au Sénégal: Une étude qualitative}

FATOU BINTOU MBOW, NAFISSATOU DIOP, AWA DIOP DABO, AWA TOUNKARA CISSE, SEYNABOU BA DIAKHATE 


\section{L'expérience de femmes ayant subi une grossesse non désirée au Sénégal: Une étude qualitative}

FATOU BINTOU MBOW', NAFISSATOU DIOP1, AWA DIOP DABO2, AWA TOUNKARA CISSE ${ }^{3}$, SEYNABOU BA DIAKHATE ${ }^{4}$

1 Population Council Sénégal

2 Réseau Siggil Jiggen (RSJ)

${ }^{3}$ Association des Juristes Sénégalaises (AJS)

${ }^{4}$ Association des Femmes Médecins du Sénégal (AFEMS) 
STEP UP œuvre en faveur d'une recherche pertinente à l'élaboration des politiques afin de promouvoir une approche basée sur des données pour améliorer l'accès à la planification familiale et à l'avortement sans risques. Nous travaillons au Bangladesh, dans la partie Nord de l'Inde, au Ghana, au Kenya et au Sénégal. STEP UP est coordonné par le Population Council en partenariat avec African Population and Health Research Center; icddr,b; London School of Hygiene and Tropical Medicine; Marie Stopes International; et Partners in Population and Development. STEP UP est financé par UKaid du Gouvernement du Royaume Uni. https://stepup.popcouncil.org

\section{POPULATION COUNCIL \\ Ideas. Evidence. Impact.}

Le Population Council s'attelle aux questions cruciales de la santé et du développement - qu'il s'agisse d'endiguer la progression du $\mathrm{VIH}$, d'améliorer la santé reproductive et d'offrir aux jeunes la promesse d'une vie satisfaisante et productive. Par ses travaux de recherche en biomédecine, en sciences sociales et en santé publique dans 50 pays, il s'efforce d'apporter avec ses partenaires des solutions qui conduisent à des politiques, des programmes et des technologies plus efficaces, pour améliorer la vie partout dans le monde. Fondé en 1952, le Population Council est une organisation non gouvernementale à but non lucratif. II siège à New York sous la conduite d'un conseil d'administration international. www.popcouncil.org

Citation recommandée: Fatou Bintou Mbow, Nafissatou Diop, Awa Diop Dabo, Awa Tounkara Cissé, Seynabou Ba Diakhaté. 2016. "L'expérience de femmes ayant subi une grossesse non désirée au Sénégal: Une étude qualitative " Rapport de recherche. STEP UP. Dakar: Population Council.

(C) 2016, Population Council

Pour plus d'informations, veuillez contacter:

Dr. Harriet Birungi, hbirungi@popcouncil.org

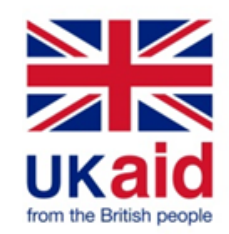




\section{Table des Matières}

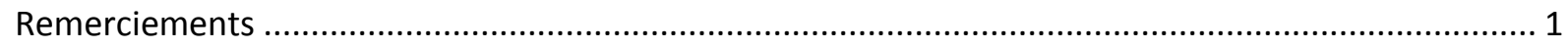

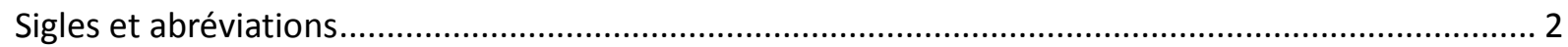

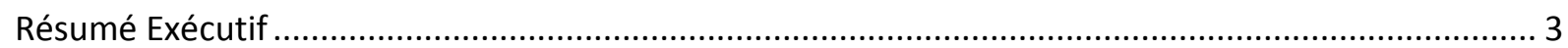

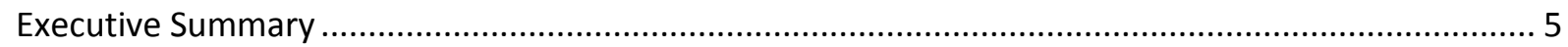

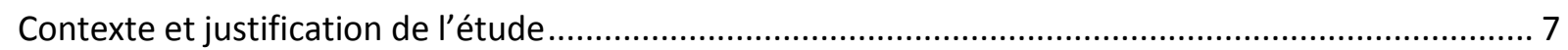

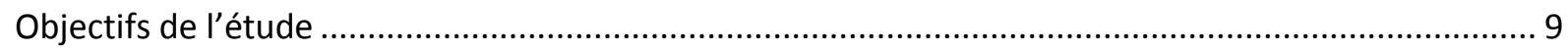

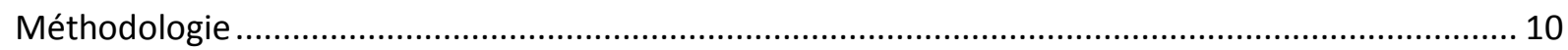

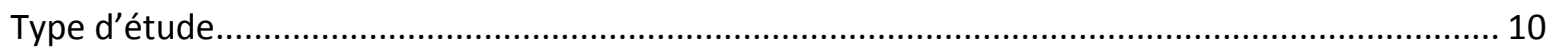

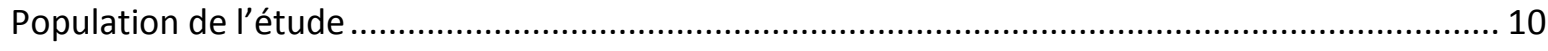

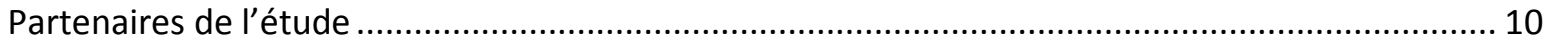

Procédure de recrutement et de collecte ....................................................................... 11

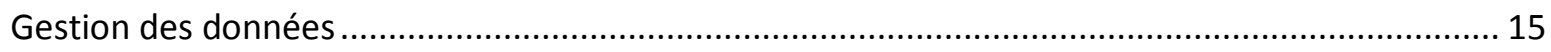

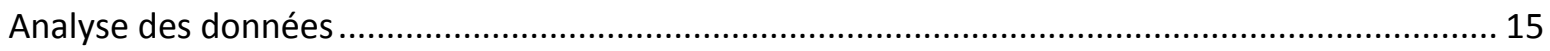

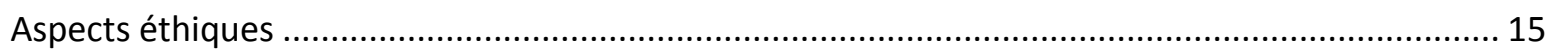

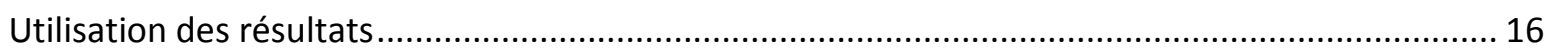

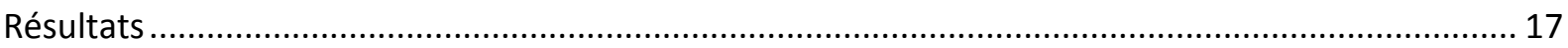

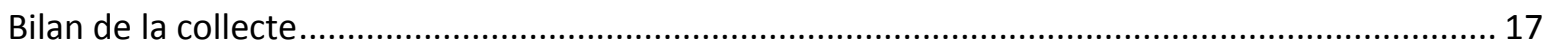

Caractéristiques sociodémographiques des populations interviewées..................................... 18

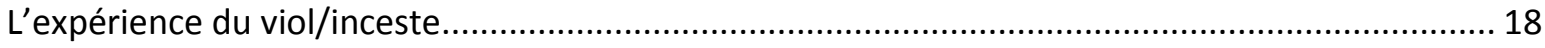

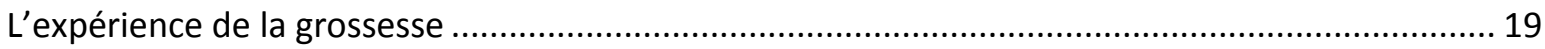

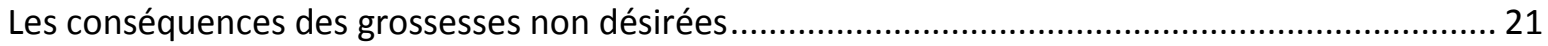

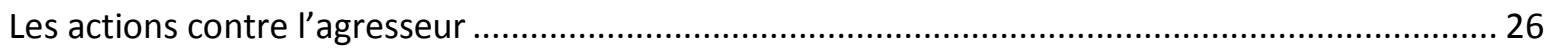

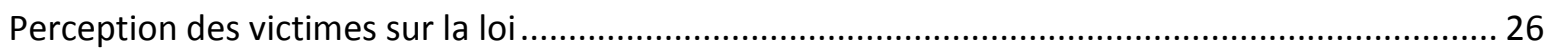

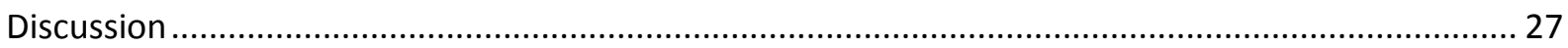

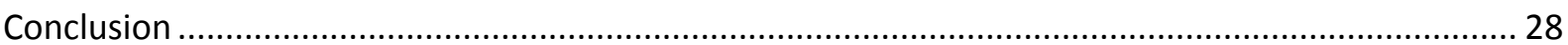

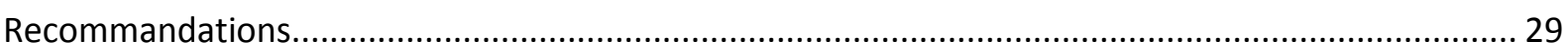

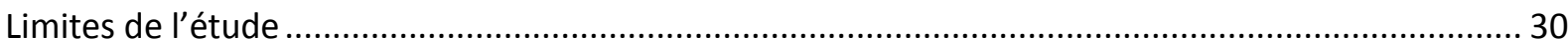




\section{Remerciements}

Cette recherche a été réalisée en collaboration avec l'Association des Femmes Médecins du Sénégal (AFEMS), l'Association des Juristes Sénégalaises (AJS) et le Réseau Siggil Jiggen (RSJ), Organisations de la Société Civile (OSC) sénégalaises et membres du Comité de plaidoyer pour l'accès à l'avortement médicalisé au Sénégal, communément appelé « La Taskforce». Ces OSC ont pris une part active dans la conception, la mise en œuvre et la publication. Les conditions parfois difficiles sur le terrain ne les ont pas empêchées de mener avec détermination les entretiens avec les victimes.

Nous remercions aussi toutes les personnes, structures, organisations et autorités qui ont, à travers leur bienveillance et leur collaboration, facilité cette recherche.

Nous exprimons aussi toute notre gratitude aux victimes qui ont bien voulu participer à la présente recherche, en acceptant de témoigner de leurs expériences et opinions, en partageant leurs souffrances et espoirs, malgré la sensibilité du sujet. Un des objectifs de cette recherche était de leur donner une voix dans le débat pour ou contre l'avortement médicalisé au Sénégal. Nous espérons, à travers les résultats de cette recherche et les recommandations formulées, contribuer à une meilleure compréhension de ce que les victimes de viols et d'incestes résultant de grossesses non désirées vivent dans leur chair et au quotidien et aider au plaidoyer pour un assouplissement de la loi sur l'avortement médicalisé et éviter de futures victimes.

Cette recherche a été réalisée grâce au soutien financier le DFID du Royaume Uni à travers le Programme de Recherche du Consortium « Strengthening Evidence for Programming on Unintended Pregnancies (STEP UP) ». Les activités de la Taskforce sont financées en partie par la Fondation Hewlett à travers un financement accordé au Population Council. 


\section{Sigles et abréviations}

$\begin{array}{ll}\text { AFEMS } & \text { Association des Femmes Médecins du Sénégal } \\ \text { AJS } & \text { Association des Juristes Sénégalaises } \\ \text { CEGID } & \text { Centre de Guidance Infantile Familiale } \\ \text { EDS } & \text { Enquête Démographique et de Santé } \\ \text { EDS-C } & \text { Enquête Démographique et de Santé Continue } \\ \text { EDS-MICS } & \text { Enquête Démographique et de Santé à Indicateurs Multiples } \\ \text { MAC } & \text { Maison d'Arrêt et de Correction } \\ \text { MAF } & \text { Maison d'Arrêt pour Femmes } \\ \text { MSAS } & \text { Ministère de la Santé et de l'Action Sociale (MSAS) } \\ \text { ONG } & \text { Organisation Non-Gouvernementale } \\ \text { OSC } & \text { Organisation de la Société Civile } \\ \text { PF } & \text { Planification Familiale } \\ \text { RSJ } & \text { Réseau Siggil Jiggen } \\ \text { SR } & \text { Santé de la Reproduction }\end{array}$




\section{Résumé Exécutif}

Au Sénégal, en 2010, environ 3.6 pour cent des décès maternels ont été liés à un avortement à risque, tandis que 51500 avortements provoqués ont été reportés en 2012. Pourtant, le Sénégal a ratifié des traités et conventions au niveau régional et international relatifs à la santé de la femme. Malgré cela, le pays a l'une des lois les plus restrictives du monde sur l'avortement. En 2013, le Comité de plaidoyer pour l'accès à l'avortement médicalisé au Sénégal (aussi appelé la Taskforce) a été créé pour mener des activités de plaidoyer pour une révision de cette loi. Depuis, l'avortement est plus ouvertement discuté. Cependant, la voix des femmes victimes de grossesse non désirée n'est pas entendue.

Dans le cadre du Projet Strengthening Evidence for Programming on Unintended Pregnancy (STEP UP), et sur financement de DFID, une étude qualitative a été menée dans la région de Dakar, au Sénégal, par le Population Council en partenariat avec la Taskforce pour développer une meilleure compréhension de la vie des femmes ayant subi une grossesse non désirée suite à un viol ou un inceste et apporter leur voix au débat sur la légalisation de l'avortement médicalisé.

La méthodologie a consisté en des entretiens approfondis (EA) réalisés en collaboration avec trois (3) Organisations de la Société Civile (OSC) membres de la Taskforce, à savoir :

Le Réseau Siggil Jigeen (RSJ), qui a mené les interviews avec des femmes qui ont subi une grossesse non désirée résultant d'un viol ou d'un inceste, ont gardé la grossesse, et l'enfant est né vivant et a survécu au moins un an (Groupe 1);

L'Association des Juristes Sénégalaises (AJS), pour les femmes qui ont subi une grossesse non désirée résultant d'un viol ou d'un inceste, ont tenté d'interrompre la grossesse ou ont commis un infanticide et ont été sanctionnées par la loi (Groupe 2); et

L'Association des Femmes Médecins du Sénégal (AFEMS) pour les femmes qui ont subi une grossesse non désirée résultant d'un viol ou d'un inceste et ayant souffert de conséquences dramatiques sur leur santé (Groupe 3).

La collecte des données s'est déroulée sur une période de deux (2) mois pour chaque groupe entre le 15 juin et le 30 août 2015 selon la date de complétude de la formation des intervieweuses.

\section{Résultats}

15 entretiens ont été complétés sur un total de 36 candidates potentielles identifiées. Le Groupe 1 a conduit neuf (9) interviews, le Groupe 2 en a conduit quatre (4), et le Groupe 3 deux (2). Concernant l'expérience du viol/inceste, pour l'ensemble des groupes, l'acte a souvent été commis par une personne connue de la victime. Beaucoup de femmes préfèrent ne pas en parler. Mais parmi celles qui décident de briser le silence, peu bénéficient de soutien/assistance de leur famille et amis.

Par rapport à l'expérience de la grossesse, les résultats montrent que pour Groupe 1, les femmes ne voulaient pas réellement garder l'enfant issu du viol/inceste, mais elles s'y sont résignées pour diverses raisons dont les croyances religieuses, la fatalité et la peur de la loi. Les conséquences de la grossesse sont la stigmatisation, la discrimination, l'échec scolaire, la honte/déshonneur de la famille, la rancœur envers les hommes, le manque d'affection vis-à-vis de l'enfant/rejet, la précarité économique, les difficultés à se (re)marier, les violences physiques par les membres de la famille, et le déni de paternité de l'auteur/fuite de responsabilité. Pour Groupe 2, les femmes déclarent n'avoir jamais envisagé de commettre un infanticide. La plupart ne veulent pas reconnaitre avoir commis un infanticide et déclarent que l'enfant était mort-né. Celles qui admettent l'infanticide avancent comme arguments la volonté d'éviter la honte, l'affolement ou la peur. Les conséquences pour ce groupe sont la détention prolongée, la stigmatisation, la perte de revenus, la rupture familiale (notamment avec leurs autres enfants) et les 
remords. Pour Groupe 3, les raisons pour celle ayant gardé la grossesse sont liées à la religion et au fatalisme, tandis que pour celle ayant fait un avortement, il s'agit de la peur et de la volonté de poursuivre ses études. Les conséquences dans les deux (2) cas sont des troubles psychologiques, l'échec scolaire, la stigmatisation et la discrimination. Les conséquences supplémentaires sont la honte/déshonneur de la famille pour celle ayant gardé la grossesse, les douleurs pelviennes pour celle ayant fait un avortement.

L'étude montre par ailleurs que très peu d'actions sont entreprises par les victimes ou leurs familles contre les agresseurs qui jouissent d'une impunité. Concernant la législation sur l'avortement, les victimes la jugent injuste, avec de nombreuses conséquences (pour les femmes) et veulent qu'elle soit modifiée.

\section{Conclusion}

La législation actuelle contribue à victimiser doublement les femmes. Qu'elles décident de garder la grossesse non désirée ou de s'en débarrasser, dans tous les cas, leur vie en est affectée. Elles font face à de graves conséquences sociales, économiques, juridiques et sanitaires. En interdisant l'avortement, notamment en cas de viol/inceste, le Sénégal « viole » des droits protégés par différents traités et conventions qu'il a pourtant ratifiés. L'adoption d'une loi permettant l'avortement médicalisé est nécessaire pour le respect des droits humains et de santé des femmes, la diminution de la prévalence, et la réduction des conséquences des grossesses non désirées et des avortements à risque pour les victimes, leur famille et la société en général. 


\section{Executive Summary}

In Senegal, in 2010, about 3.6 percent of maternal deaths were related to unsafe abortions while 51,500 induced abortions were reported in 2012. Yet, Senegal has ratified treaties and conventions at regional and international levels related to women's health. Nevertheless, the country has one of the most restrictive law on abortion in the world. In 2013, the Advocacy committee for access to safe abortion in Senegal (also called the Taskforce) was created to conduct advocacy for the revision of that law. Since then, abortion is more openly discussed. However, the voice of women victims of unwanted pregnancy is not heard.

As part of the Strengthening Evidence for Programming on Unwanted Pregnancies (STEP UP) Project, and with funding from DFID, a qualitative study was conducted in the Dakar region, in Senegal, by the Population Council in partnership with the Taskforce to develop a better understanding of the lives of women who had an unwanted pregnancy resulting from rape or incest and bring their voices to the debate on the legalization of safe abortion.

The methodology consisted of In-depth Interviews (IDI) conducted in collaboration with 3 Civil Society Organizations (CSO) members of the Taskforce, that are:

- The Siggil Jigeen Network (RSJ), that conducted the interviews with women who had an unwanted pregnancy resulting from rape or incest, kept the pregnancy, and the child was born alive and survived for at least one year (Group 1);

- The Senegalese Women Lawyers Association (AJS), for women who had an unwanted pregnancy resulting from rape or incest, attempted to end the pregnancy or committed an infanticide and were sanctioned by the law (Group 2 ); and

- The Association of Senegalese Women Medical Doctors (AFEMS), for women who had an unwanted pregnancy resulting from rape or incest, and suffered dramatic health consequences (Group 3).

Data collection took place over a 2-month period for each group between June 15 and August 30, 2015, depending of interviewers' training completion date.

\section{Results}

15 interviews were completed out of 36 potential women identified. Group 1 conducted 9 interviews, Group 2 did 4 , and Group 32 . Regarding the experience of rape/incest, for all the groups, the act was often committed by someone known to the victim. Many women prefer not to talk about it. However, among those who decide to break the silence, few receive support/assistance from their family and friends.

Regarding the experiences of pregnancy, results show that for Group 1, women did not really want to keep the child born out of the rape/incest, but they gave in for various reasons, including religious beliefs, fate and fear of the law. The consequences of the pregnancy are stigmatization, discrimination, school failure, shame/family dishonor, resentment towards men, lack of affection for the child/rejection, no financial support, difficulties in (re) marry, physical violence by family members, and denial of paternity of the author/evades responsibility. For Group 2, women claim never considering committing an infanticide. Most of them do not want to acknowledge committing infanticide and declare that the child was stillborn. Those who admit committing an infanticide explain it by the desire to avoid shame, panic or fear. Consequences for this group are prolonged detention, stigmatization, loss of income, family disruption (especially with their other children) and remorse. For Group 3, the one who kept the pregnancy gave reasons related to religion and fatalism, while for the one who had an abortion it was because of fear and the desire to continue her education. In both cases, consequences are psychological issues, school failure, stigmatization and discrimination. Additional consequences are shame/family dishonor for the one who kept the pregnancy, pelvic pain for the one who have an abortion. 
The study also shows that very few actions are taken by the victims or their families against the perpetrators who enjoy impunity. Regarding legislation on abortion, victims consider it unfair, with many consequences (for women) and want it changed.

\section{Conclusion}

The current law contributes to victimizing women twice. Whether they decide to keep the unwanted pregnancy or to eliminate it, either way, their life is changed forever. They face serious social, economic, legal and health consequences. By prohibiting abortion, in particular in case of rape/incest, Senegal "violates" rights protected by treaties and conventions it had nevertheless ratified. The adoption of a law allowing safe abortion is required for the respect of women's human and health rights, the decrease in prevalence and consequences of unwanted pregnancies and unsafe abortions to victims, their family, and the society as a whole. 


\section{Contexte et justification de l'étude}

Au Sénégal, le taux élevé de mortalité maternelle pose un réel problème de santé publique et de développement. II est estimé à 392 cas de décès pour 100000 naissances en 2010-2011, d'après l'Enquête Démographique et de Santé Continue (EDS-C) de 2014. Chaque jour, cinq (5) femmes meurent en donnant la vie, parmi elles, celles victimes de complications des avortements clandestins dus aux grossesses non désirées. Une évaluation des grossesses non désirées et des avortements à risque au Sénégal menée par IPAS en 2010 pour le Ministère de la Santé reporte qu'environ 3,6 pour cent des décès maternels étaient liés aux avortements à risque1. Ces avortements à risque représentaient environ la moitié de toutes les admissions d'urgence vers les services de santé maternelle. Plus récemment, selon les estimations de l'étude de l'Institut Guttmacher réalisée en partenariat avec le Centre de Recherche pour le Développement Humain (CRDH)², 51500 avortements ont été provoqués en 2012 au Sénégal. Et toujours selon la même source, 38 pour cent de ces avortements ont été effectués par un prestataire traditionnel ou autre prestataire non qualifié.

La loi sur la Santé de la Reproduction (SR) a été adoptée en 2005. Cependant, elle dispose en son article 15 que "L'interruption volontaire de grossesse est interdite sauf lorsqu'elle est autorisée par la loi. En aucun cas, elle ne saurait être considérée comme une méthode contraceptive». Ainsi, au Sénégal, l'avortement n'est autorisé que lorsque la vie de la mère est menacée (Article 35 du code de déontologie médicale ${ }^{3}$ ). Cette restriction favorise le recours aux avortements à risque.

Pourtant, le Sénégal a ratifié plusieurs lois et traités internationaux concernant la santé de la femme. Ceux-ci incluent le Protocole de la Charte Africaine des Droits de l'Homme et des Peuples relatif aux Droits des Femmes en Afrique, mieux connu sous le nom du Protocole de Maputo. Ce protocole a été adopté par l'Union Africaine le 11 juillet 2003 lors de son deuxième sommet à Maputo (Mozambique). Il est entré en vigueur le 25 novembre 2005. Dans son article 14, le protocole dispose que « Les États assurent le respect et la promotion des droits de la femme à la santé y compris la santé sexuelle et reproductive». Ceci inclut le fait de « protéger les droits reproductifs de la femme en autorisant l'avortement médicalisé en cas d'agression sexuelle, de viol, d'inceste, et lorsque la grossesse met en danger la santé mentale et physique de la mère ou la vie de la mère ou du fœtus ». Le Sénégal a ratifié le protocole de Maputo le 8 janvier 2005 et publié au Journal officiel sous le $n^{\circ} 6200$.

En 1985, le Sénégal a également ratifié la Convention sur l'élimination de toutes formes de discrimination à l'égard des femmes (CEDEF), qui garantit en son article 12 le droit des femmes d'accéder aux services médicaux, y compris aux services de planification familiale (PF). En juillet 2015, le Comité d'experts de la CEDEF a recommandé de modifier l'article 305 du Code pénal, le Code de déontologie médicale, ainsi que d'autres dispositions juridiques pertinentes relatives à la dépénalisation de l'avortement et, veiller à ce que l'avortement soit légalement disponible en cas de danger ou menace pour la vie ou la santé de la femme enceinte, en cas de viol, d'inceste et de déficience grave du fœtus. Ce comité d'experts a également recommandé de retirer du projet de réforme du Code pénal les dispositions juridiques mettant à la charge des femmes enceintes le fardeau de la preuve que leur grossesse résulte d'un viol ou d'un inceste.

1 Ministère de la Santé et de la Prévention. Division de la Santé de la Reproduction. Rapport de l'évaluation stratégique sur les grossesses non désirées et les avortements à risque au Sénégal, Mai 2010.

2 Institut Guttmacher, Centre de Recherche pour le Développement Humain (CRDH). L'incidence et la morbidité de l'avortement provoqué au Sénégal. 2015

3 Décret $N^{\circ} 67-147$ du 10 février 1967 
Malgré cela, le Sénégal a encore l'une des lois les plus restrictives sur l'avortement dans le monde. L'article $35 \mathrm{du}$ Code de déontologie médicale prévoit une unique exception qui indique que : «ll ne peut être procédé à un avortement thérapeutique que si cette intervention est le seul moyen susceptible de sauvegarder la vie de la mère». De plus, cette exception, très limitée, est accompagnée de conditions procédurales extrêmement lourdes et onéreuses. En effet, trois (3) médecins différents (le médecin prescripteur et deux (2) autres médecins dont l'un est expert auprès des tribunaux) doivent attester que la vie de la mère ne peut être sauvée qu'au moyen d'une telle intervention. Ensuite un protocole de la décision prise doit être adressé sous pli recommandé au Président de l'Ordre des Médecins. Cependant, « Si le médecin, en raison de ses convictions, estime qu'il lui est interdit de conseiller de pratiquer l'avortement, il peut se retirer en faisant assurer la continuité des soins par un confrère qualifié ». Soumis à des règlements aussi radicaux et interminables, très peu de gens peuvent répondre à ces exigences, et en temps opportun.

De plus, la législation sénégalaise considère l'avortement provoqué comme une infraction pénale. Et les dispositions des articles 305 et 305 bis du Code pénal prévoient contre leurs auteurs des peines d'emprisonnement allant de six (6) mois à trois (3) ans et/ou des amendes de 50.000 à un million de FCFA.

Devant toutes ces barrières, de nombreuses filles et femmes désespérées ont recours à l'infanticide ou à des avortements à risque, le plus souvent accompagnés de conséquences sociales, économiques, juridiques, psychologiques et sanitaires. Parfois même, elles y perdent la vie. Des chiffres officiels ${ }^{4}$ montrent qu'au cours des six (6) premiers mois de l'année 2013, un quarantaine de femmes avaient été placées en détention provisoire pour avoir pratiqué un infanticide ou une interruption volontaire de grossesse ${ }^{5}$.

En 2012, des députés avaient déjà tenté de dépénaliser l'avortement en soumettant à l'Assemblée Nationale une proposition de loi. Celle-ci avait rencontré la vive opposition de la plupart des politiciens sénégalais, symbolisant le tabou que représente encore la question de l'avortement.

C'est dans ce contexte qu'en 2013, les activités du Comité de plaidoyer pour l'accès à l'avortement médicalisé au Sénégal (plus communément appelé « Taskforce ») ont été relancées sous l'égide du Ministère de la Santé et de l'Action Sociale (MSAS). La Taskforce est un comité pluridisciplinaire composée d'institutions étatiques, d'organisations de la société civile, et d'organisations non gouvernementales (ONG). II mène des activités visant à informer les décideurs politiques et religieux, les medias, et la population en général sur la problématique de l'avortement à risque, notamment en cas de viol ou d'inceste. Ainsi, avec l'assistance de leaders religieux, et de spécialistes dans les domaines juridique, sociologique, économique et sanitaire, la Taskforce a développé une brochure intitulée «Accès à l'avortement médicalisé au Sénégal. Réalités, défis et mythes ». D'autres supports de communication ont aussi été produits pour aider à mieux toucher les différentes parties prenantes. Le plaidoyer a déjà permis que la question de l'avortement médicalisé au Sénégal soit plus facilement débattue dans l'espace publique.

Un des objectifs de la Taskforce est l'élaboration de propositions de réforme de textes, notamment le code pénal, le code de déontologie médicale et la loi SR, pour y inclure l'ensemble des cas énumérés par le Protocole de Maputo dans lesquels l'avortement médicalisé devrait être autorisé. Cependant, les propositions n'ont pas encore été adoptées par le Conseil des Ministres. La Taskforce continue son plaidoyer.

4 Rapport semestriel 2013 de la Direction de l'administration pénitentiaire.

5 II est difficile d'avoir les statistiques séparées de l'avortement et de l'infanticide parce que la Division de la législation des Statistiques et de l'Instruction de la Direction de l'Administration Pénitentiaire fait remarquer qu'ils ne font pas toujours la différence entre l'avortement et l'infanticide. 
Par ailleurs, si l'on se base sur le rapport FIDH/RADDHO/LSDH (2014), d'autres acteurs à différents niveaux se sont mobilisés pour l'adoption du projet de réforme de ces textes. Ainsi, plusieurs femmes députés se sont attelées à sensibiliser les parlementaires encore réticents et travaillent pour l'adoption de ces textes. Le Ministère de la Justice, a également mis en place des ateliers d'information à l'attention des groupes parlementaires. Toutefois, le gouvernement ne s'est pas encore prononcé clairement sur son soutien à la réforme.

Malgré ces différentes actions, la voix des victimes n'est pas suffisamment entendue. C'est dans la perspective de comprendre le vécu des filles/femmes victimes de viol et d'inceste et d'ajouter leur point de vue au débat que le Population Council a entrepris la présente étude en partenariat avec la Taskforce. En effet, entendre les histoires racontées avec leurs propres mots par les victimes pourrait contribuer à faire prendre conscience de leur réalité, et aider à développer des arguments et des messages de plaidoyer pour un assouplissement de la loi sur l'avortement, en mettant un visage humain sur l'impact des grossesses non désirées et des avortements illégaux sur les victimes de viol et d'inceste, leurs enfants, leurs familles et sur la société en générale. Cela pourrait également amener le Sénégal à harmoniser ses textes avec les conventions et instruments internationaux qu'il a ratifiés.

\section{Objectifs de l'étude}

L'objectif général de cette étude est de développer une meilleure compréhension de la vie des femmes qui ont subi une grossesse non désirée suite à un viol ou un inceste.

Les objectifs spécifiques sont de :

documenter la vie des femmes qui ont subi une grossesse non désirée résultant d'un viol ou d'un inceste et qui n'ont pas interrompu la grossesse (Groupe 1);

documenter la vie des femmes qui ont été reconnues coupables d'avortement illégal ou d'infanticide et qui ont été sanctionnées par la loi (i.e. les femmes qui ont été incarcérées suite à cet acte) (Groupe 2) ;

- documenter la vie des femmes dont l'état de santé s'est dramatiquement dégradé en raison d'une grossesse suite à un viol ou l'inceste (Groupe 3); et

fournir des évidences basées sur les recherches à la Taskforce et à tous ceux qui sont intéressés par le sujet pour servir dans les activités de plaidoyer pour un assouplissement de la loi sur l'avortement. 


\section{Méthodologie}

Pour mettre en lumière la situation des femmes ayant subi une grossesse non désirée suite à un viol ou un inceste, et plus particulièrement les conséquences auxquelles elles font face, une approche participative a été utilisée.

\section{Type d'étude}

II s'agit d'une étude qualitative conduite dans la région de Dakar.

\section{Population de l'étude}

L'étude avait pour cible les femmes ayant subi une grossesse non désirée résultant d'un viol ou d'un inceste et âgées de 18 ans ou plus au moment de l'interview. Cette cible a été scindée en trois (3) groupes:

Groupe 1: Femmes ayant subi une grossesse non désirée résultant d'un viol ou d'un inceste, qui n'ont pas interrompu la grossesse et l'enfant est né vivant et a survécu au moins un (1) an. Cette durée de survie de l'enfant permettait d'éliminer les cas de décès néonatals dus à d'autres causes. En outre, l'enfant aurait vécu suffisamment longtemps pour pouvoir constater l'effet que cet enfant aurait pu avoir sur la vie de la mère et de ceux qui l'entourent.

- Groupe 2: Femmes ayant subi une grossesse non désirée résultant d'un viol ou d'un inceste, ont tenté d'interrompre la grossesse ou ont commis un infanticide, et ont été sanctionnées par la loi. Les femmes pouvaient être incarcérées ou avoir purgé leur peine.

Groupe 3: Femmes ayant subi une grossesse non désirée résultant d'un viol ou d'un inceste et ayant souffert de conséquences dramatiques sur leur santé. Dans ce groupe ont été inclues les familles de femmes décédées des conséquences médicales d'une grossesse non désirée suite à un viol ou un inceste.

\section{Partenaires de l'étude}

Trois (3) organisations de la société civile (OSC), membres de la Taskforce, ont été associées à l'étude. Ces OSC sont:

Le Réseau Siggil Jigeen (RSJ). Cette ONG vise à promouvoir et à protéger les droits de la femme au Sénégal. Le réseau est composé de 16 organisations membres qui s'efforcent à améliorer la vie de plus de 12000 femmes sénégalaises à travers diverses initiatives telles que celles liées au statut de la femme, à la reproduction et à la santé, à la recherche, aux droits de la femme, au leadership des jeunes, à l'alphabétisation, à la formation, à la microfinance et à la lutte contre la pauvreté. Pour cette étude, RSJ a été impliquée dans les entretiens avec des femmes du Groupe 1, c'est-à-dire les victimes qui ont décidé de ne pas interrompre leur grossesse.

L'Association des Juristes Sénégalaises (AJS) regroupe des femmes juristes de toutes branches de droit (juges, avocates, inspectrices de travail, conseillères juridiques, étudiantes en droit, etc.) du secteur public et privé. L'AJS œuvre pour la vulgarisation, la promotion des droits humains, plus particulièrement ceux des femmes et des enfants. Pour cette étude, l'AJS a été impliquée dans la réalisation d'entretiens avec des femmes du Groupe 2 - c'est-à-dire les victimes qui ont commis un acte illégal et ont été condamnées. Par convention avec le système juridique au Sénégal, l'AJS a accès aux femmes en prison et a mené des enquêtes avec elles auparavant. De ce fait, avec cette étude, l'AJS n'en était pas à sa première expérience pour mener des entretiens de ce genre avec les détenues. En outre, l'AJS pouvait avoir gardé le contact avec les femmes qui ont purgé leurs peines et se sont réintégrées dans la communauté. 
L'Association des Femmes Médecins du Sénégal (AFEMS). Cette association professionnelle de femmes médecins participe pleinement à l'amélioration de la santé des populations en général, celle des femmes, des jeunes, des enfants et des personnes les plus démunies en particulier. Elle s'engage en outre dans différents domaines parmi lesquels la SR avec la lutte contre la mortalité maternelle et néo-natale, et la lutte contre les violences faites aux femmes et aux enfants et leur prise en charge. L'AFEMS a été impliquée dans la réalisation des entretiens avec des femmes du Groupe 3, c'est-à-dire les victimes dont la santé s'est dramatiquement dégradée suite à une grossesse résultant d'un viol ou d'un inceste. Dans certains cas, l'AFEMS pouvait également interviewer la famille des femmes qui avaient perdu la vie à la suite d'un avortement sur une grossesse due au viol ou à l'inceste.

\section{Procédure de recrutement et de collecte}

\section{Outils de collecte}

Cinq (5) outils de collecte ont été développés pour servir de support à des entretiens individuels approfondis, à savoir :

Guide d'entretien avec les femmes qui ont subi une grossesse non désirée résultant d'un viol ou d'un inceste, qui ont poursuivi la grossesse et l'enfant est né vivant et a vécu au moins un (1) an ;

- Guide d'entretien avec les femmes qui ont subi une grossesse non désirée résultant d'un viol ou d'un inceste, qui ont tenté d'interrompre la grossesse et sont actuellement incarcérées ;

- Guide d'entretien avec les femmes qui ont subi une grossesse non désirée résultant d'un viol ou d'un inceste, ont tenté d'interrompre la grossesse, ont été incarcérées, et qui sont actuellement libres ;

- Guide d'entretien avec les femmes qui ont subi une grossesse non désirée résultant d'un viol ou d'un inceste et ayant souffert de conséquences dramatiques sur leur santé ;

- Guide d'entretien avec la famille des femmes qui ont subi une grossesse non désirée résultant d'un viol ou d'un inceste et qui n'ont pas survécu.

Les outils développés ont été basés sur des sujets d'intérêt pour la Taskforce. Ils ont permis de collecter des informations sur les expériences des femmes relativement au viol et à la grossesse, leurs relations avec la famille/l'entourage, les conséquences du viol /de l'inceste et les actions prises contre l'agresseur. Les questions ont été pré-testées et les guides d'entretiens ont été finalisés en collaboration avec les OSC.

\section{Profil des intervieweuses}

Compte tenu de la nature sensible du sujet, tous les agents de collecte étaient des femmes. Elles avaient, pour quelques-unes, un minimum d'expérience dans la conduite d'enquêtes dans le domaine de la santé.

Afin de faciliter l'identification des cibles, et tenant compte de l'hypothèse que les participantes seraient familières avec les OSC partenaires de l'étude (RSJ, AJS et AFEMS) ou leurs activités, il a été retenu que les intervieweuses soient des membres de l'OSC chargée du groupe spécifique. Cette approche devait aussi permettre de contribuer à préserver l'anonymat des participantes, à les mettre plus à l'aise et à mieux garantir la confidentialité.

Pour chaque OSC, le Population Council et I'OSC ont sélectionné trois (3) intervieweuses et une (1) coordinatrice de l'étude. Les intervieweuses parlaient couramment le français et le wolof, la langue locale la plus communément parlée dans la région de Dakar. Elles ont travaillé sous la supervision et avec le soutien de l'équipe de recherche du Population Council. 


\section{Formation des équipes des OSC}

Chaque OSC a reçu une formation en recherche qualitative individualisée et adaptée aux spécificités de son groupe. Dans chaque cas, les intervieweuses et la coordonnatrice ont été formées ensemble pour renforcer la cohésion du groupe et assurer une compréhension commune de leurs tâches.

Un préalable à l'étude a été de doter les participantes d'une bonne compréhension des principes et méthodes de la recherche qualitative, du procédé à suivre dans le choix des femmes et des autres informateurs, et de la collecte des données. Un accent particulier a été mis sur les considérations éthiques, notamment le caractère volontaire de la participation et l'utilisation des formulaires de consentement. La deuxième partie de la formation a été consacrée à l'étude approfondie des outils de collecte.

La formation a aussi insisté sur la rigueur requise pour recueillir les informations, et s'assurer qu'autant les intervieweuses que les coordonnatrices ont une bonne compréhension de l'étude et du procédé de recrutement et de collecte pour leur groupe.

Pendant les sessions, les instruments de collecte ont été verbalement traduits en wolof. En outre, les exercices de simulation d'interviews ont permis de mieux assimiler les instruments. Ces derniers ont ensuite été prétestés et les modifications nécessaires ont été apportées pour les clarifier et les finaliser.

\section{Procédure de recrutement et de collecte des données}

Pour chaque OSC, en dehors des intervieweuses chargées de collecter les données, une coordinatrice a été désignée pour l'étude. Les entretiens approfondis ont été conduits selon le schéma suivant :

Groupe 1: Femmes qui ont subi une grossesse non désirée résultant d'un viol ou d'un inceste, qui n'ont pas interrompu la grossesse et l'enfant est né vivant et a survécu au moins un (1) an.

1. RSJ a fait appel à ses 13 animatrices communautaires $(A C)$, dont certaines sont également des Bajenu Gox, réparties dans la région de Dakar. Les AC sont en contact direct avec les communautés dans lesquelles elles travaillent et mènent des activités liées à la promotion de bonnes pratiques et de comportements liés à la santé. Les AC ont utilisé leur bonne connaissance des événements liés à la santé des membres de la communauté, telles les grossesses, pour identifier les cas éligibles dans leurs communautés. Les AC ont bénéficié de l'appui de délégués de quartiers et d'autres collaborateurs (ex : encadreurs dans des clubs de jeunes, directeurs de collectivités éducatives).

2. RSJ a aussi mis à contribution certaines structures telles que la Maison Rose, le Centre Guindi, le CEGID.... qui s'activent dans l'encadrement et la prise en charge des victimes de violences basées sur le genre.

3. Pour chaque cas identifié, la coordinatrice a vérifié que la femme répondait effectivement aux critères d'éligibilité.

4. L'AC ou le représentant de la structure d'assistance, selon le cas a contacté la femme afin de lui expliquer l'étude et lui demander si elle est intéressée à participer. Pour chaque femme qui a accepté de participer, la personne-contact a informé la coordinatrice.

5. la coordinatrice a organisé une rencontre entre une intervieweuse du RSJ formée et la femme. Pour chaque participante, l'entretien a été mené dans le lieu de son choix.

6. une fois en présence de la femme consentante, l'intervieweuse a commencé par expliquer de nouveau l'étude et demander son consentement éclairé. 
Groupe 2: Femmes ayant subi une grossesse non désirée résultant d'un viol ou d'un inceste, ont tenté d'interrompre la grossesse ou ont commis un infanticide, et ont été sanctionnées par la loi. Les femmes pouvaient être incarcérées ou avoir purgé leur peine.

Pour ce groupe, les femmes éligibles ont été identifiées et abordées de 2 manières :

Pour les femmes détenues:

1. Une lettre officielle a été adressée par l'AJS à la directrice de la Maison d'Arrêt pour Femmes (MAF) de Liberté VI afin d'introduire l'étude et demander sa collaboration dans l'identification des candidates éligibles. Ensuite la directrice a été contactée directement par la coordonnatrice de l'étude.

2. Grâce à la collaboration de la greffière, les fiches des détenues ont été triées. Au moment de l'enquête, les trois (3) femmes qui étaient accusées d'avortement venaient juste d'être transférées dans une autre MAF. De ce fait, seules les fiches des détenues accusées d'infanticides ont pu être triées (27 au total). Une rencontre a été organisée avec chacune de ces détenues pour vérifier les critères d'éligibilité (femme ayant plus de 18 ans, incarcérée pour infanticide (dans ce cas-ci), et dont la grossesse a été contractée suite à un viol ou un inceste), expliquer l'étude à celles qui étaient éligibles, et leur demander si elles étaient intéressées à participer.

3. Pour celles qui ont accepté de participer, des rencontres ont été organisées avec l'appui de la greffière entre ces femmes et des intervieweuses d'AJS formées. Les entretiens ont été réalisés dans les limites de la prison dans une salle polyvalente choisie par les autorités pénitentiaires.

4. Une fois en présence d'une femme consentante, l'intervieweuse a commencé par lui expliquer de nouveau l'étude et demandé son consentement éclairé.

Pour les femmes sorties de prison, plusieurs pistes ont été exploitées.

1. Des messages pour expliquer l'étude ont été envoyés à tous les membres de l'AJS, aux avocats partenaires ou membres de l'AJS qui ont eu à défendre de tels cas.

2. Une lettre de demande d'assistance a été envoyée à l'ONG Tostan qui s'active notamment dans la réinsertion sociale et professionnelle des anciennes détenues.

3. Une ancienne détenue a été également mise à contribution.

Groupe 3: Femmes ayant subi une grossesse non désirée résultant d'un viol ou d'un inceste et ayant souffert de conséquences dramatiques sur leur santé. Ce groupe incluait aussi les familles des femmes décédées des conséquences sanitaires d'une grossesse non désirée suite à un viol ou un inceste.

Pour ce groupe, L'AFEMS a été impliquée dans la réalisation des entretiens. Le processus a été le suivant :

1. L'AFEMS a informé ses membres de l'étude pour aider à l'identification des cibles.

Elle a également sollicité d'autres personnes-contacts, y compris des confrères médecins appartenant aux associations d'obstétriciens/ gynécologues, des sages-femmes, la Maison rose et le CEGID.

2. Pour chaque cas identifié, la coordinatrice de l'étude a été contactée pour vérifier que la personne répondait effectivement aux critères d'éligibilité.

3. La personne éligible a été contactée par la personne-contact pour recevoir des explications sur l'étude et pour savoir si elle était intéressée à y participer. 
4. Pour chaque personne qui a accepté de participer, la personne-contact en a informé la coordinatrice qui a organisé une rencontre entre la personne et une intervieweuse AFEMS formée. Pour chaque participante, l'entretien a été mené dans le lieu de son choix.

5. Une fois en présence d'une personne consentante, l'intervieweuse a commencé par expliquer l'étude de nouveau et demander son consentement éclairé.

\section{Collecte des données}

Pour chaque groupe, la collecte des données s'est effectuée durant 2 mois. Les OSC ont commencé au fur et à mesure que leur formation se terminait, d'où le léger décalage observé entre la période de collecte de RSJ (Groupe 1) et AFEMS (Groupe 3).

Compte tenu de la possibilité qu'une participante pouvait ne pas vouloir parler de (toute) son expérience dès la première interview, il a été prévu jusqu'à trois (3) rencontres par participante. Cela, afin de lui donner du temps pour se sentir à l'aise et parler à son propre rythme. Chaque séance a duré 1 heure maximum. Par ailleurs, une liste de contacts utiles (professionnels, organisations spécialisées) a été compilée à partir des références de chaque OSC pour permettre aux participantes qui le désirent de recourir, à leur frais, à une aide psychologique, juridique ou médicale, quand elles se sentent prêtent pour le faire.

L'équipe de recherche du Population Council n'a pas eu de contact direct avec les femmes. Cependant, elle a mené une supervision très rapprochée des intervieweuses et de la qualité des entretiens effectués.

Des réunions de bilan périodiques ont été organisées avec les coordonnatrices au niveau des OSC pour faire le point sur l'état d'avancement de la collecte, apporter les réponses aux problèmes soulevés, et assister lorsque nécessaire pour le bon déroulement de la collecte.

\section{Difficultés rencontrées}

Quelques difficultés ont été notées à savoir :

Problème d'identification des participants : plusieurs des personnes identifiées au départ étaient des mineures et ne répondaient donc pas au critère d'âge.

La sensibilité du sujet et le manque de confiance ont conduit au refus de certaines personnes de participer à l'étude. Elles n'étaient pas prêtes psychologiquement pour un récit. De plus, les femmes éligibles ainsi que leurs familles voulaient oublier cet épisode de leur vie. En outre, les organisations de défense des droits des femmes gèrent le plus souvent des cas récents de viol et d'inceste dont les victimes ne sont pas non plus prêtes à participer à de telles études.

Dans certains cas, essentiellement au niveau de la prison, le cadre n'était pas toujours approprié pour garantir la confidentialité des interviews. Par exemple, la salle mise à la disposition des intervieweuses était une salle polyvalente où les détenues se regroupent habituellement pour mener leurs différentes activités. II fallait donc à chaque fois négocier avec les autorités pénitentiaires pour trouver d'autres occupations aux autres détenues afin de permettre à l'intervieweuse de s'isoler avec la femme. De plus, les rencontres étaient conditionnées à la disponibilité de la greffière. Par exemple, lorsqu'un rendez-vous était fixé avec la femme et que pour une quelconque raison la greffière devait s'absenter, le rendez-vous était annulé, même si cette dernière ne devait pourtant pas être physiquement présente à l'entretien.

L'ambiance souvent triste et pleine d'émotion des entretiens a parfois été dure à supporter aussi bien par les interviewées que par les intervieweuses, même dans les cas où les faits remontaient à 20 ans ou plus. 
L'incompréhension/la confusion de certaines femmes incarcérées des objectifs de l'étude. Lors de l'identification des cibles, certaines ont nourri des espoirs, pensant que leur participation les aiderait par rapport à leur dossier judiciaire. II a fallu plusieurs fois bien recadrer les objectifs de l'étude.

Le manque d'accès direct aux victimes pour l'équipe de recherche du Population Council. Les entretiens ont été menés par des personnes qui n'étaient pas des chercheurs (notamment dans le domaine de la recherche qualitative), même si certaines avaient une expérience en matière d'enquête. Aussi, il a fallu mettre un accent particulier sur la formation des OSC en recherche, sur le suivi pour le respect des procédures éthiques et scientifiques, et sur la qualité des données.

\section{Gestion des données}

Les entretiens individuels ont été enregistrés dans la langue choisie par l'interviewée, puis transcrits par les intervieweuses (en français si l'interview avait été conduit en wolof) à l'aide de Microsoft Word. Pour garder l'anonymat et la confidentialité des participantes, les informations qui permettraient de les identifier et de les localiser ont été codées. Par ailleurs, les données ont été archivées sous format électronique. Les transcriptions anonymisées ainsi que les fichiers audio des entretiens ont été conservées dans les ordinateurs du bureau de Population Council protégés par mots de passe conformément aux critères de protection des données du Population Council. Seule l'équipe de recherche peut accéder aux données.

\section{Analyse des données}

L'analyse de contenu a été utilisée pour l'interprétation des données recueillies. Elle a combiné une démarche descriptive et une démarche inductive, en vue de présenter un état des lieux.

\section{Aspects éthiques}

\section{Autorisations éthiques}

Le protocole de recherche a été soumis pour approbation au Comité d'éthique du Population Council et au Comité National d'Ethique pour la Recherche en Santé (CNERS) du MSAS du Sénégal. Les avis favorables de ces deux (2) institutions ainsi que l'autorisation administrative de mise en œuvre de l'étude délivrée par la Direction de la Planification, de la Recherche et des Statistiques du MSAS ont été officiellement obtenus.

\section{Consentement éclairé}

Le consentement éclairé écrit a été sollicité et obtenu pour chaque personne interviewée. Les participantes ont été informées sur les conditions de participation à l'étude, les objectifs, les risques et bénéfices et les procédures de collecte. Elles ont toutes décidé de participer en toute connaissance de cause et en toute liberté.

\section{Confidentialité et anonymat}

La protection et le respect de la confidentialité et de la vie privée des interviewées a été un aspect essentiel de cette étude. Afin de protéger l'anonymat des femmes et les aider à se sentir plus à l'aise à participer à une telle étude, les procédures suivantes ont été mises en place :

Les femmes ont été identifiées par les OSC à travers leurs activités professionnelles. Pour maintenir l'anonymat de ces femmes, il a été convenu que ce seront des membres de ces OSC qui feront les contacts et les interviews. Ainsi, pour chaque OSC, quatre (4) membres ont été formés à cet effet. De plus, lors de la formation des OSC, un accent particulier a été mis sur le respect des procédures éthiques.

Pour préserver l'intimité visuelle et auditive des femmes, les entretiens, en dehors de ceux menés dans la prison, se sont déroulés dans le lieu de leur choix avec la possibilité de s'isoler avec l'enquêtrice. 
- Pour s'assurer de l'anonymat des répondants et des informations collectées, aucune information sur l'identité n'a été enregistrée sur les notes d'entretien, ni sur les enregistrements, ni sur les transcriptions électroniques. Les données ont été gardées séparément des identifiants.

- L'accès aux données électroniques et aux fichiers audio des entretiens stockés dans les locaux du bureau du Population Council a été sécurisé et limité à l'équipe de recherche. Les ordinateurs ont été protégés par mots de passe.

- Les résultats des entretiens avec les femmes sont présentés de façon agrégés afin que les réponses ne puissent pas être directement reliées aux individus ou aux sites. Pour les cas uniques, toute information qui permettrait l'identification de la personne a été enlevée.

\section{Compensation}

Aucune compensation financière n'a été versée pour toute participation à l'étude. Cependant, conformément au protocole de recherche, pour les participantes, les frais de transport aller/retour (pour un maximum de 3 rencontres) vers et du lieu de l'interview ont été remboursés. Les femmes détenues, pour lesquelles les entretiens n'ont pas nécessité de déplacements, ont reçu à la place des effets de toilette, conformément à la pratique de l'AJS lors des visites en prison.

\section{Utilisation des résultats}

Les résultats de cette étude seront utilisés par la Taskforce et largement partagés avec d'autres parties prenantes pour contribuer à des efforts de plaidoyer étayés par des données probantes, et pour faire entendre la voix des victimes dans les débats juridiques, politiques, sociaux et religieux autour de la légalisation de l'avortement médicalisé dans le pays. 


\section{Résultats}

\section{Bilan de la collecte}

Le tableau ci-dessous donne un aperçu du bilan de la collecte.

Tableau 1 : Bilan de la collecte

\begin{tabular}{|c|c|c|c|c|}
\hline & & Groupe ci & SC & \\
\hline & Groupe 1 & Groupe 2 & Groupe 3 & \\
\hline & RSJ & AJS & AFEMS & Total \\
\hline Période de collecte & $\begin{array}{l}15 \text { Juin- } 30 \text { Août } \\
2015\end{array}$ & $\begin{array}{l}\text { 03 Juin-30 Août } \\
2015\end{array}$ & $\begin{array}{l}13 \text { Juillet- } 30 \text { Août } \\
2015\end{array}$ & \\
\hline $\begin{array}{l}\text { Nombre total de femmes potentielles } \\
\text { identifiées }\end{array}$ & 18 & 13 & 5 & 36 \\
\hline $\begin{array}{l}\text { Nombre de femmes identifiées, mais } \\
\text { ayant refusé de participer à l'étude }\end{array}$ & 9 & 7 & 3 & 19 \\
\hline Nombre d'entretiens complétés & 9 & 4 & 2 & 15 \\
\hline Nombre d'entretiens incomplets & 0 & 2 & 0 & 2 \\
\hline
\end{tabular}

Au total, sur les 36 personnes potentielles identifiées, 15 femmes ont complété leurs entretiens, à savoir neuf (9) pour le Groupe 1, quatre (4) pour le Groupe 2 et deux (2) pour le Groupe 3.

Le refus de participer a été plus manifeste dans les Groupes 2 et 3 , bien que toutes les OSC reportent que cela est essentiellement lié au désir des femmes de ne plus vouloir se remémorer ces moments terribles de leur vie.

Pour le Groupe 2 :

Concernant les 13 femmes potentielles qui ont été identifiées (8 femmes incarcérées et 5 ex-détenues), la situation se présentait de la manière suivante :

Pour ce qui est des femmes incarcérées, seules des détenues accusées d'infanticide (27 au total) ont pu être retrouvées avec l'assistance de la greffière. Trois (3) femmes qui étaient accusées d'avortement venaient d'être transférées dans une autre Maison d'arrêt et de correction (MAC) hors de la zone de l'étude. Sur les 27 femmes, 8 étaient réellement éligibles après vérification. Cependant, deux (2) ont refusé de participer. La raison de refus est que ces femmes ont fait des amalgames entre les objectifs de l'étude et leur situation en prison et ont craint d'aggraver leur cas en témoignant. Des six (6) restantes, quatre (4) ont accepté de participer et ont complété leur interview. Les deux (2) autres femmes, ont accepté de participer à l'étude, mais n'ont pas pu terminer leur interview. Pour ces entretiens non complétés, les raisons sont essentiellement liées aux critères d'éligibilité. En effet, bien que les femmes aient déclaré avoir été victimes de viol, les intervieweuses se sont rendues compte en cours d'entretien que la grossesse n'était pas réellement issue d'un viol ou d'un inceste. Cela a immédiatement conduit à l'interruption de l'entretien.

Pour les ex-détenues, cinq (5) candidates potentielles n'ont pas voulu participer à l'étude suite à un antécédent avec les médias où, au cours d'une interview dans le cadre d'une activité avec d'autres intervenants, des victimes qui tenaient à leur anonymat ont quand même pu être identifiées. Hormis ces femmes, aucune autre ayant déjà purgé sa peine n'a pu être identifiée et interrogée. Ceci est entre autres dû au fait que certains cas potentiels étaient des migrantes venues chercher du travail dans la capitale et elles étaient retournées au village.

Pour le Groupe 3, aucun proche de femmes décédées n'a pu être identifié dans les délais impartis. 


\section{Caractéristiques sociodémographiques des populations interviewées}

\section{Groupe 1}

Pour ce groupe, les interviewées étaient âgées entre 18 et 43 ans. Ce groupe compte six (6) célibataires, deux (2) mariées et une (1) divorcée. Elles sont toutes musulmanes. On compte parmi elles des sans-emploi (4), des élève/étudiante (2), une femme de ménage, une commerçante et une employée d'usine. L'âge de leurs enfants se situe aujourd'hui entre 1 et 26 ans.

\section{Groupe 2}

Dans ce groupe, les quatre (4) femmes interviewées sont âgées entre 24 et 37 ans. On compte deux (2) célibataires, une (1) divorcée et une (1) mariée. Elles sont toutes musulmanes. En dehors de l'une d'entre elles qui n'a jamais travaillé, les trois (3) autres sont d'anciennes domestiques. Elles sont toutes actuellement incarcérées pour infanticide.

\section{Groupe 3}

Pour ce groupe, les deux (2) personnes interrogées sont âgées de 20 et 37 ans. Elles sont célibataires et musulmanes. L'une est commerçante, tandis que l'autre tente de reprendre ses études. L'une a gardé la grossesse et l'autre a effectué un avortement. Elles souffrent aujourd'hui toutes les deux de troubles psychologiques, voire même psychiatriques pour l'une. Celle ayant tenté un avortement souffre en plus de douleurs pelviennes.

\section{L'expérience du viol/inceste}

Pour tous les groupes, les femmes interviewées déclarent avoir été victimes de viol à l'exception d'une (parmi celles qui ont gardé l'enfant) qui affirme avoir été victime d'inceste de la part de son oncle.

Pour la majorité, il s'agit d'acte commis par des personnes connues, à savoir un voisin, un ami de la famille, l'excopain, le marabout, etc. Deux tiers des cas de viol relatés par les participantes l'ont été avec violences physiques. Pour le tiers restant, les femmes déclarent avoir été violées dans des circonstances où elles se trouvaient dans l'incapacité de contrôler la situation ou de se défendre car elles affirment avoir été droguées suite à l'absorption de liquides suspects. Le témoignage qui suit en est une parfaite illustration.

« Je voulais consulter un voyant qui pourrait m'aider afin que je puisse avoir la paix avec ma mère. Quand j'ai expliqué mon problème à une voisine, elle m'a dit qu'elle connaissait un marabout avec qui elle m'a mis en rapport. C'est ainsi que j'y suis allée pour une séance de voyance munie de 500 francs CFA. II m'a promis de me faire du "SAFARA " [liquide béni] que je viendrai chercher un autre jour. Et le jour où je suis venue récupérer le "SAFARA » il m'a violé.... II m'a remis le "SAFARA » j'en ai bu un peu sur place, et ensuite j'ai enduit le "SAFARA » sur mon corps et j'ai eu l'impression de dormir et c'est là qu'il a abusé de moi. Quand je me suis réveillée je lui ai dit : “qu'est-ce que tu m'as fait ?"II m'a répondu je ne t'ai rien fait, tu as seulement dormi » (37 ans, détenue pour infanticide).

Aussitôt après le viol/inceste, beaucoup de femmes se sont empressées de faire disparaître toute trace de l'agression notamment en se lavant, en nettoyant l'endroit où l'acte a eu lieu, ou en cachant les vêtements qu'elles portaient. Elles ont pour la plupart préféré ne pas en parler à leur entourage, famille ou proches, par honte ou crainte de la réaction de ces derniers ou de la réaction de l'agresseur comme en témoigne cette femme violée par un agent de police: « Je n'en ai parlé à personne parce que j'avais peur que l'agresseur ne m'emprisonne. Parce que le jour du drame il m'a menacé de me balancer en prison, raison pour laquelle je ne l'ai révélé à personne ». Dans de nombreux cas, la révélation du viol par les femmes coïncide avec la découverte de la grossesse par les proches. Pour les rares personnes qui en ont parlé aussitôt, très peu ont bénéficié du soutien (moral, accompagnement...) de l'entourage familial. La réaction des parents, dans beaucoup de cas était le plus souvent répressive, certaines se voyant même accusées d'être à l'origine de leur drame. C'est ainsi qu'une jeune fille de 
18 ans aujourd'hui mère de jumeaux affirme : « Un de mes oncles disait que je l'avais fait exprès, que c'est moi qui l'ai voulu car les autres filles ont des maris. Je n'avais plus d'habits, il m'a violemment frappée parce que j'avais emprunté des vêtements à une amie, ensuite il m'a déshabillée car pour lui, j'ai humilié la famille ».

\section{L'expérience de la grossesse}

Les conditions dans lesquelles les grossesses non désirées suite au viol/inceste sont vécues sont très pénibles pour les victimes quel que soit leur groupe.

\section{Groupe 1}

Aucune des femmes interrogées ne souhaitait réellement garder l'enfant issu du viol/inceste. Elles se sont résignées à le faire pour plusieurs raisons :

- L'existence de croyances religieuses : A l'instar de la société sénégalaise en général, beaucoup de femmes interrogées perçoivent l'avortement comme un interdit religieux.

- La fatalité : Dans certains cas, la survenue de la grossesse, quel qu'en soit les circonstances est considérée comme un signe du destin, une volonté divine qui doit être acceptée comme telle. De ce fait, certaines femmes n'ont pas eu d'autre choix que de la garder.

- La peur de la loi : Même si beaucoup de femmes ne maitrisent pas le contenu de la loi, certaines savent que l'avortement est une infraction et que les femmes qui y recourent encourent une peine de prison.

- Le stade avancé de la grossesse au moment de sa découverte : Certaines femmes ont compris assez tard qu'elles étaient enceintes. Quand elles l'ont découvert, la grossesse était âgée de 3, voire 4 mois. Terme à partir duquel l'idée de s'en débarrasser présentait des risques pour ellesmêmes.

- La volonté des proches : Pour certaines femmes et filles enceintes, c'est l'opposition de l'entourage familial qui a constitué un obstacle à l'avortement, les obligeant à garder leur grossesse.

- La peur des conséquences sanitaires. Si certaines femmes n'ont pas eu recours à l'avortement clandestin, c'est par peur des risques sanitaires que cette pratique pourrait présenter. 
L'encadré suivant montre des exemples de propos recueillis.

« II faut laisser l'enfant naître parce que en le tuant tu auras deux péchés et ce n'est pas facile de se faire pardonner deux péchés donc c'est un danger » (24 ans, employée d'usine)

" Je me disais toujours que je ne vais pas me laisser perturber, je ne dirais rien qui pourrait salir sa réputation, et j'ai considéré que cela devait peut être m'arriver» (24 ans, commerçante)

« Mon père m'a dit " si tu avortes, je t'amène à la police....Tu n'es pas la première et tu ne seras pas la dernière, ce n'est que la volonté de Dieu » (19 ans, élève)

"Ma mère voulait qu'on enlève la grossesse pour ne pas perturber mes études, mais mon grand-père a dit non. Le père va le reconnaître ou on ira en justice, mais l'enfant va naître » (19 ans, ménagère)

"Ce n'est pas bien, c'est des risques même si c'est un fœtus, si tu avortes tu peux perdre beaucoup de sang ou détruire ton utérus, tu peux ne plus avoir d'enfants ou tu risques la prison. J'ai déjà vu des cas comme ça » (24 ans commerçante)

Même si certaines femmes reconnaissent avoir bénéficié du soutien sur le plan économique, moral ou affectif de parents, proches ou amis durant cette période, ce n'est pas le cas pour la majorité d'entre elles qui ont dû faire face à de nombreuses difficultés. Elles se retrouvent très souvent livrées à elles-mêmes, sans aucun recours ou aide pour la prise en charge de leurs besoins primaires. Non seulement les relations avec leur entourage deviennent tendues, voire conflictuelles, mais en plus, elles doivent supporter le regard des autres.

\section{Groupe 2}

Pour cette catégorie, les femmes pensent et affirment avoir mené leur grossesse à terme dans la discrétion la plus totale. En effet, elles ont toutes tenté de la dissimuler de peur, pour certaines, d'affecter leurs proches comme en témoignent les propos de cette ancienne domestique de 24 ans : " Je ne voulais pas faire souffrir ma mère, elle aurait été déçue, j'avais laissé tomber les études pour l'aider, pas pour m'amuser. Si elle apprenait ma grossesse elle serait accablée ". Elles pensaient avoir tellement bien caché leur grossesse qu'elles n'ont pas toujours réussi à expliquer comment elles ont été découvertes, hormis l'une d'entre elles qui déclare avoir été dénoncée par l'auteur de la grossesse.

Si elles ont choisi de cacher leur grossesse, c'est surtout pour avoir nourri, reconnaissent-t-elles, l'idée durant toute cette période, de se débarrasser de l'enfant à la naissance, non pas en le tuant, mais en le plaçant dans une pouponnière ou en l'offrant à des personnes. Toutes tiennent à préciser qu'elles n'ont jamais envisagé durant toute leur grossesse de commettre un infanticide.

Par ailleurs, même si certaines participantes ne veulent toujours pas reconnaître le fait d'avoir commis un infanticide dans la mesure où, selon elles, elles auraient donné naissance à un mort-né, quelques-unes assument leur acte. Cependant elles tiennent à préciser que c'est seulement après l'accouchement qu'elles ont décidé de se débarrasser de l'enfant en mettant fin à sa vie. Plusieurs raisons ont été avancées pour justifier leur démarche.

Pour éviter la honte : Beaucoup de femmes ne souhaitaient pas jeter l'opprobre sur elle-même ou sur leur famille.

L'affolement: Juste après l'accouchement, les femmes déclarent avoir été désemparées et prises de panique. Une situation qui les aurait conduites à passer à l'acte.

La peur : Peur de la réaction du mari, mais aussi peur d'être la risée de l'entourage (famille, amis, voisins).

« Une chose me faisait peur : mon époux....qu'il me trouve avec un enfant dont il n'est pas le père. Je me disais qu'il m'a abandonné pendant cinq ans. Cet enfant conçu et né indépendamment de ma volonté, je vais le garder s'il nait vivant. Mais c'est le jour de l'accouchement que ce mauvais esprit m'est venu....Je ne pensais qu'à mon 
époux pour qu'il ne me crée pas encore de problèmes. J'avais peur aussi de la famille....lls peuvent attendre un bon jour et te cracher tout sur la figure. Tout ceci pouvait arriver » (32 ans, ménagère).

\section{Groupe 3}

L'étude a révélé 2 situations :

1) La victime porteuse de grossesse non désirée, dès lors qu'elle s'est rendue compte qu'elle était enceinte, n'a trouvé comme solution que le recours à l'avortement, clandestin puisque prohibé, par peur de la réaction des parents, et pour éviter de déshonorer la famille, mais aussi pour pouvoir terminer ses études.

« J'ai pensé à avorter parce que mon père ... il est célèbre, donc après il y a tout ce problème de scandale politique... il fallait tout enterrer. Je me suis dit c'est une affaire de honte etc... il fallait enlever ça parce que c'est un problème de réputation ...Si un journaliste était au courant, ça aurait eu un effet sur mon père et ça c'était un problème à éviter » (20 ans, étudiante).

2) La victime a décidé de mener la grossesse à terme pour être en phase avec les recommandations religieuses lorsqu'une telle circonstance se présente, et surtout parce qu'elle considérait cette grossesse comme un signe du destin.

«C'était mon destin, quelque chose qui devait m'arriver et qui est arrivé subitement...c'est Dieu qui l'a voulu ainsi, c'est pour cela que c'est arrivé » (37 ans, commerçante).

Dans les deux (2) cas, la grossesse non désirée a été à l'origine de problèmes de santé que les femmes tentent de surmonter jusqu'à ce jour.

\section{Les conséquences des grossesses non désirées}

Les grossesses non désirées suite au viol/inceste exposent les femmes à de nombreuses conséquences. Certaines sont similaires, bien que des nuances soient marquées selon le groupe. D'autres sont spécifiques à chaque groupe. Cette section présente les conséquences rapportées par groupe.

\section{Groupe 1}

\section{Stigmatisation}

Alors que certaines femmes affirment avoir été soutenues par leur entourage compte tenu des circonstances dans lesquelles la grossesse est survenue, l'étude a révélé qu'il est arrivé souvent que plusieurs d'entre elles aient été stigmatisées par la famille, les amis ou les voisins. Cette stigmatisation pouvait prendre plusieurs formes, telles que : être injuriée ou pointée du doigt, ou faire l'objet de commérages. Toutes les occasions étaient bonnes pour leur rappeler ce qui s'était passé. Certaines ont dû faire face à l'interdiction par des voisins de continuer à fréquenter leurs enfants.

Au-delà de la nature, l'étude donne également des informations sur l'étendue de cette stigmatisation qui peut se prolonger jusqu'à la maman ou même à l'enfant. Au Sénégal, un adage dit: « Quand un enfant réussit, c'est l'enfant de tout le monde, mais quand c'est un déviant, c'est l'enfant de sa maman », ce qui sous-entend que c'est la faute à sa maman. Cette dernière en subit les conséquences. Par ailleurs, dans l'ethnie Ouolof, il existe une expression pour désigner l'enfant né hors mariage. C'est un doomu xaraam [enfant du péché], une expression qui est très révélatrice de la place qu'il occupe dans la communauté. Il est considéré comme le dernier de la société. 
L'encadré ci-après présente quelques propos de victimes qui traduisent l'acuité de ces situations:

" J'étais fatiguée. Je n'osais plus sortir, j'ai tout entendu surtout du côté de la famille de mon père. C'était très difficile, on me disait "thiagabi" [la traînée/la prostituée] et toutes sortes de mauvaises paroles" (22 ans, ménagère).

"Ils disaient : “celle-là, son enfant n'a pas de père "et cela me faisait mal » (42 ans, femme de ménage).

"Après l'accouchement, c'était plus difficile car je croyais que tout allait changer, mais ma mère m'a fait comprendre que si je mettais les pieds à la maison, je pouvais briser son ménage » (24 ans, employée d'usine).

" Ma mère n'a pas voulu en parler à mon père pour sauvegarder son mariage, mais c'est ma grand-mère qui a parlé à mon père... et il a commencé à ignorer ma mère » (24 ans, commerçante).

" Tu sais, il [l'enfant] ne se laisse jamais pousser les cheveux... parce que quand il passe devant les maisons, on le taquine en lui disant " naar nga ! naar nga ! [Tu es un maure ! tu es un maure !] ». C'est pour cela qu'il se rase tout le temps » (42 ans, femme de ménage).

« II m'était très difficile de sortir. Les voisins sont allés jusqu'à interdire à leur enfants avec qui j'étais tout le temps de me fréquenter car ils disaient que j'ai trainé jusqu'à ce que ce qui m'est arrivé me soit arrivé » (22 ans, ménagère).

\section{- Discrimination}

Les informations recueillies ont permis de relever plusieurs cas de discrimination que subissent certaines victimes de la part de leur famille, amis ou voisins. Ces pratiques discriminatoires se manifestent par : le manque de conseils ou de soutien (économique, affectif...) de sa famille contrairement aux autres membres, l'exclusion de certaines activités familiales (cérémonies, repas....) ou par le fait d'être ignorée et marginalisée.

" Ils [les parents] ne m'aident pas. J'ai une ordonnance qui s'élève à $5500 \mathrm{~F}$ mais je ne peux pas la payer, elle est là. Je suis fatiguée, et une mère de jumeaux ne peut pas travailler » (18 ans, ménagère).

« Je n'avais plus personne à qui parler. Je restais toute seule, c'est peut-être cette solitude qui m'avait poussée à dire qu'après l'accouchement j'allais donner mon enfant à quelqu'un» (24 ans, employée d'usine).

" Je ne recevais plus de conseils de leur part. Ils m'avaient mise en quarantaine, j'étais exclue de tout ce qu'ils faisaient surtout du côté de mon père et des autres membres de la famille» (28 ans, étudiante).

«Cela n'a pas plu à ma mère ....Elle ne peut plus me supporter. Ma mère ne me soutient en rien. Tout ce que je lui dis, elle me rétorque qu'elle s'en fiche. Elle ne m'accorde plus la moindre importance. Elle ne sait rien de moi .....Mes petites sœurs et frères aussi ne parvenaient pas à supporter que je sois enceinte. J'étais mariée, mais je n'ai pas eu d'enfant dans ce mariage. Le fait d'avoir un enfant après le divorce est insupportable pour eux jusqu'à présent. Comme je viens de le dire personne ne veut m'aider » (43 ans, ménagère).

\section{- Honte/ Gêne}

Dans beaucoup de cas, le fait de porter une grossesse non désirée suite à un viol ou un inceste plonge les victimes dans la honte et crée le malaise vis-à-vis de leurs parents, amis ou voisins.

" Jusqu'à présent “"kersa bi "[la gêne/le complexe] est là, et même quelques fois quand j'ai besoin d'acheter un slip ou des serviettes hygiéniques, quand je ne travaille pas, j'ai des problèmes. Ma mère, elle veut m'aider, mais elle aussi ne peut pas. Quand elle me dit demande à ton père, je dis non, parce-que ma dignité ne me permet plus de lui demander quoi que ce soit » (24 ans, employée d'usine).

« Une fois à l'hôpital, on m'a posée des questions et demandé à ma tante si jeétais mariée. Elle a eu honte et a dit oui. Ils ont demandé à ma tante "est-ce que son mari est là"? ... elle a dit « son mari a voyagé ». (24 ans, commerçante) 


\section{- Rancœur}

Suite à leur expérience, certaines femmes en veulent définitivement aux hommes et leur vouent une haine incommensurable. Plus grave, des tendances dépressives et parfois suicidaires, ont été constatées chez certaines.

« J'avais une douleur profonde, J'ai pleuré, j'ai détesté les hommes, et jusqu'à présent, je déteste les hommes, les hommes vraiment j'en ai assez et si ce n'était pas ma mère j'allais me suicider» (28 ans, étudiante)

« Je suis actuellement mariée, même si je ne l'aime pas. Mais j'étais prête à me marier avec toute personne que ma mère me proposerait car je sais tout ce qu'elle a subi à cause de moi, mais je ne voulais plus entendre parler des hommes. Même quand j'ai des rapports sexuels avec mon mari, je n'éprouve aucun plaisir car je déteste les hommes » (22 ans, ménagère).

\section{- Déshonneur de la famille}

A la suite de ce qu'elles ont vécu, certaines femmes ont été accusées par leurs proches d'avoir déshonorer leur famille au point de faire l'objet de violences physiques de leur part.

" Je veux de l'aide pour mes jumeaux, ils sont anémiés. Je mendiais tous les jeudis et vendredis, mais les membres de ma famille me battaient toujours sous prétexte que j'humilie toute la famille » (18 ans, ménagère).

\section{Manque d'affection vis-à-vis de l'enfant}

Etant donné que pour ce groupe toutes les femmes ont maintenu leur grossesse malgré elle, certaines reconnaissent que leur enfant souffre de manque d'affection de leur part car il est le témoin vivant de leurs souffrances passées. Les propos de cette étudiante dont l'enfant est aujourd'hui décédé en témoigne : « Je ne l'ai jamais aimé. Quand je le voyais, je me rappelais de ce qui m'était arrivé. C'était la seule personne que je ne voulais pas voir. Je ne voulais pas le voir » (28 ans, étudiante).

« Les conséquences sont nombreuses, moi j'ai maintenant le cœur dur, tellement je souffre. Je n'ai plus pitié. Tu veux même mourir. Parfois, j'en veux à mon fils, bien qu'il ne soit pas fautif » (43 ans, ménagère)

\section{- Déni de paternité}

Beaucoup d'agresseurs ne veulent pas reconnaitre les grossesses, encore moins les enfants à leur naissance. Ce refus de reconnaissance de paternité fait partie des conséquences les plus douloureuses à vivre pour les victimes. Elles l'assimilent d'ailleurs à une fuite de responsabilité.

\section{Précarité socio-économique}

Suite à leur expérience, beaucoup de femmes vivent dans des situations précaires. Elles n'ont pas d'aide pour leur prise en charge. Elles se trouvent dans l'incapacité de subvenir à leurs besoins, encore moins à ceux de leur enfant.

\section{- Difficultés à se (re)marier}

Plusieurs femmes ont fait remarquer qu'elles font rarement l'objet de demande en mariage. Elles pensent que si jusqu'à présent elles n'arrivent pas à se marier ou à se remarier, c'est à cause de ce qu'elles ont vécu. Certes, la société sénégalaise désapprouve les naissances hors mariage, mais pour elles, les circonstances assez particulières dans lesquelles ces enfants sont nés constituent un obstacle supplémentaire à leur (re)mariage. 


\section{- Déscolarisation}

Les grossesses non désirées ont eu des incidences négatives sur la scolarisation de certaines filles. En effet, comme l'ont montré les résultats de l'étude, ces grossesses ont conduit à des échecs ou à l'abandon de la scolarité. Et après la naissance de leur enfant, beaucoup de victimes ne manifestent plus un intérêt pour leurs études. $\mathrm{Ce}$ qui apparait dès lors comme un obstacle majeur à leur éducation. Vu que beaucoup d'entre elles ne bénéficient plus du soutien de leur famille, il leur est souvent difficile d'allier en même temps la prise en charge de l'enfant et la poursuite des études.

\section{Groupe 2}

\section{Détention prolongée}

Des femmes sont aujourd'hui emprisonnées/poursuivies par la justice pour avoir eu recours à l'infanticide alors que le Code Pénal le réprime. En fait, si l'on se base sur les articles 285, 287 et 289, elles risquent la peine de mort ou les travaux forcés à perpétuité. Cette interdiction légale contribue à créer l'isolement autour d'elles.

\section{- Stigmatisation}

Au même titre que les femmes ayant gardé l'enfant, les femmes incarcérées souffrent de stigmatisation. Une stigmatisation qui est essentiellement fondée sur leur statut civique.

\section{- Abandon/Rupture familiale (notamment avec les enfants)}

La plupart des femmes se disent rejetées, voire abandonnées, par leurs familles, amis, voisins. Le plus dur à surmonter reste la rupture d'avec leurs autres enfants, pour celles qui en ont.

« Je n'ai aucun parent qui est venu me rendre visite ... je ne sais pas si on m'a abandonné ...Tout ce que j'ai ce sont les autres [détenues] qui me le donnent. Je me dis la manière dont je me sens si seule, dépaysée, abandonnée, j'ai peur que mes enfants ne vivent dans ces mêmes conditions. Puisque ma famille a pu me faire ça à moi, à plus forte raison mes enfants que j'ai laissés derrière moi. Tout ceci me préoccupe trop, trop. Je suis préoccupée » (32 ans, ménagère).

\section{Remords}

Sur la base des propos recueillis, il apparait que les victimes vivent désormais avec beaucoup de regrets tout ce qui leur est arrivé, et compte sur la clémence de la justice pour se réinsérer un jour dans la société.

\section{Groupe 3}

\section{- Stigmatisation}

Comme pour les autres groupes, ces femmes sont également stigmatisées. Dans certains cas, c'est à la limite si on ne les tient pas responsables de ce qui leur est arrivé.

« Rien que ce matin elle [sa maman] m'a rappelé cette histoire, rien que ce matin elle m'a rappelé cette grossesse. Tout le temps, tout le temps, c'est comme ça parce qu'elle, elle est rancunière, raison pour laquelle je ne voulais pas lui en parler... c'est chaque jour... elle me le rappelle. Une grossesse, c'est une grossesse. C'est grave, mais si ma mère n'était pas comme elle est en ce moment si elle avait réagi autrement ça aurait été beaucoup plus 
simple... Voilà quoi ! Parce que là j'ai vécu ça, mais c'est comme si je le vis tous les jours, chaque jour quand je me réveille je revis la même chose parce que je sais que ma mère me regarde avec cet œil » (20 ans, étudiante).

\section{- Honte/Déshonneur de la famille}

Compte tenu du fait que l'expérience de ces femmes crée la honte et le déshonneur pour certaines familles, ces dernières n'hésitent pas à les éloigner pour préserver leur dignité.

« Moi, mon père est un grand marabout. Quand je suis tombée enceinte, ma mère m’a emmené ailleurs pour me cacher. Je suis restée là-bas avec une tante qui me surveillait.» (37 ans, commerçante).

\section{- Conséquences sanitaires}

Ces conséquences se sont manifestées par des troubles psychologiques et des douleurs pelviennes en plus dans un cas.

« J'étais à 4 mois et demi de grossesse, presque 5...Le problème ce n'était pas la grossesse, mais l'avortement parce que là quand même j'ai senti une vie... j'ai vu les pieds sortir donc c'est un être humain, c'est ça qui m'a le plus choquée.... Après l'avortement, ma gynéco m'a mis en relation avec une psy....pour l'avortement je me suis dit dans cette expérience-là, c'est la partie la plus difficile, la plus douloureuse.... J'ai vu des pieds, un visage, des mains sortir de moi, c'est grave quoi !.... moi dans ma tête c'est pas au viol que je pense le plus, peut-être c'est moi qui suis comme ça, mais c'est à l'avortement que je pense tout le temps....quand on vit une expérience douloureuse on y repense, moi quand je pense expérience douloureuse c'est à l'avortement que je pense pas au viol, quand j'étais assise là sur la chaise anglaise, c'est à ça que je pense plus qu'au viol....La grossesse c'est un truc qui vous arrive, mais l'avortement c'est un truc que vous avez provoqué c'est quand même tuer un être humain qui aurait pu naitre et qui aurait pu devenir quelqu'un» (20 ans, étudiante).

" Moi, depuis cette histoire, je suis malade...je me fais suivre en psychiatrie. Bon c'est vrai que ça va maintenant un peu mieux, mais les problèmes que j'ai vécu, ainsi que ma maladie, je ne souhaite pas que mon enfant vive la même chose...moi je ne vais pas lui montrer un visage qui va le laisser penser que je ne vais pas bien, de peur que cela ne le perturbe » (37 ans, commerçante).

\section{- Echec scolaire}

Pour ce groupe également, l'échec scolaire a été au centre des préoccupations. Pour celle qui tente de reprendre ses études, elle est restée au moins deux (2) années successives sans pouvoir le faire. 


\section{Les actions contre l'agresseur}

L'étude montre que dans beaucoup de cas, les auteurs de viol/inceste jouissent d'une impunité totale. Pour paraphraser L. Joinet (Joinet, 1997) l'impunité ne se définit pas ici simplement comme l'absence de peine mais également comme l'absence de mise en cause de la responsabilité des auteurs.

En effet, il ressort des entretiens approfondis que très peu d'actions ont été entreprises par les victimes ou leurs familles contre les agresseurs. La plupart des actions pour les femmes qui n'ont pas interrompu leur grossesse se résument à une demande de reconnaissance de paternité et une demande de prise en charge de l'enfant. Ce qui se termine dans de nombreux cas par un refus.

Rares sont les femmes ou familles qui pensent à déposer une plainte. Et lorsque les auteurs sont arrêtés, ils bénéficieraient, aux dires de certaines victimes, de la complaisance d'agents de police.

Pour certaines victimes, notamment celles d'inceste, c'est la peur de détruire les relations familiales [ « bagne tass mbokk »] qui pourrait justifier leur absence de réaction. Cependant, pour la plupart, la disparition de l'auteur du viol/inceste, les représailles potentiels, le fait que l'agresseur soit inconnu, la peur de la stigmatisation, voire l'existence de préjugés, sont autant d'obstacles qui les empêchent ainsi que leur familles de faire recours à la justice. Les propos suivants en sont une illustration : « Des personnes l'ont suggéré à ma mère [de porter plainte], mais elle a dit que comme elle a des enfants qui sont jeunes, elle préfère ne pas le faire parce que cette situation peut aussi leur arriver » (19 ans, élève).

Par ailleurs, la difficulté à réunir les preuves nécessaires est apparue comme un frein à la saisine de la justice.

\section{Perception des victimes sur la loi}

II ressort de l'étude que près de la moitié des femmes ont connaissance de l'existence d'une loi qui interdit l'avortement, même si elles n'en maîtrisent pas le contenu.

La majorité pense qu'il s'agit d'une loi injuste, avec beaucoup de conséquences pour les femmes, et que, par conséquent, elle devrait être modifiée : "On doit changer la loi dans la mesure où en cas de viol, tu peux te retrouver avec un enfant sans père. Donc mieux vaut interrompre la grossesse. Si je prends mon cas, si une telle loi était appliquée je pouvais en bénéficier en ce moment sinon on peut avoir une grossesse non désirée et tuer l'enfant, et à ce moment c'est nous même qui allons subir les conséquences de l'infanticide ». (22 ans, ménagère). Cette autre déclare: "Pour moi, dès lors que tu n'es pas à l'origine de ce qui t'arrive, on doit te permettre de t'en débarrasser [de la grossesse], ce qu'il y a de pire c'est de garder cette grossesse, si ton enfant te demande après son père, qu'est-ce que tu vas lui répondre ? II peut même arriver qu'en grandissant elle (si c'est une fille) rencontre son père et se marie avec lui parce que c'est des situations qui peuvent arriver » (24 ans, ancienne domestique).

Les victimes ont ainsi très majoritairement plaidé pour une révision de la loi, à l'instar de cette jeune fille de 18 ans, ménagère et mère de jumeaux qui souligne que "Cette loi n'est pas bien. On devait faire de telle sorte qu'une femme victime de viol puisse enlever la grossesse ». Et une autre affirme : "Nous demandons tout simplement qu'ils [les autorités judiciaires] nous aident et nous pardonnent au nom de Dieu pour que ceci ne nous arrive plus jamais. Qu'ils nous aident au nom de Dieu, seulement au nom de Dieu, au nom de Dieu pour que cette loi qui nous est appliquée soit levée par pitié pour que nous puissions aller retrouver nos enfants pour que ce qui nous est arrivé ne leur arrive pas à eux...» (32 ans, ménagère).

L'accès à l'avortement médicalisé apparait pour certaines comme la solution idéale pour éviter certaines conséquences, notamment celles dramatiques sur la santé physique et surtout mentale. Cela a été une demande fortement exprimée. Comme l'une des victimes le mentionne : "L'avortement, ça s'est passé dans la salle de bain 
de mes parents. J'aurai préféré que ça se passe dans un hôpital là je me dis que si c'était le cas, ça m'aurait moins hanté comme c'est le cas en ce moment » (20 ans, étudiante).

\section{Discussion}

L'étude jette une lumière crue sur les drames successifs que vivent les femmes ayant subi une grossesse non désirée suite à un viol ou un inceste.

Après avoir subi un viol /inceste, qu'elles se croient dans beaucoup de cas contraintes de cacher, elles doivent supporter une grossesse non désirée. Qu'elles en gardent le produit ou décident de s'en débarrasser, dans tous les cas, leur vie en est affectée. Elles ont à faire face à de graves conséquences sociales, économiques, juridiques et sanitaires. L'interdiction de l'avortement a même conduit certaines femmes à commettre un infanticide. Leurs familles ne sont pas non plus à l'abri des conséquences de cette situation qui les affectent également.

La législation sénégalaise actuelle relative à l'avortement contribue à les victimiser doublement : d'abord en raison de l'acte subi (viol/inceste), et ensuite en raison de la stigmatisation dont elles font l'objet de la part de leur entourage. La persistance de facteurs socio-culturels véhiculant des stéréotypes et préjugés négatifs sur les femmes renforce par ailleurs la discrimination à leur égard.

Cette étude révèle la persistance des besoins en information des proches et en protection des victimes. Elle confirme également que le comportement des proches, et de la société dans son ensemble, constitue un argument supplémentaire pour renforcer le plaidoyer pour le changement de la loi, et au-delà, mener des actions de sensibilisation de la communauté pour un meilleur accompagnement des victimes. Par ailleurs, l'étude souligne l'impunité pour les auteurs de viol/inceste qui sont rarement inquiétés.

La question de la révision de la loi demeure très marquée par une forte demande également exprimée par les victimes. A cet égard, le vécu, mais également les perceptions, opinions et attentes des victimes, exprimées de manière aussi directe permettent une compréhension réaliste à la fois de l'acuité et de l'urgence de la question. La présente étude en est l'illustration. 


\section{Conclusion}

L'approche qui a consisté à mettre en avant la voix des victimes laisse apparaître la nécessité de porter une attention particulière à leurs perceptions et sur leurs rudes parcours. En prohibant l'avortement en cas de viol/inceste, le Sénégal enfreint le droit à la vie, à la santé et à l'éducation des filles, que protègent différents pactes et conventions ratifiés par le Sénégal. II s'agit notamment du Protocole de Maputo; du Pacte international relatif aux droits civils et politiques; du Pacte international relatif aux droits économiques, sociaux et culturels ; de la Convention relative aux droits de l'enfant ; et la CEDEF. Le Sénégal doit aller jusqu'au bout de son engagement proclamé pour la protection des droits des femmes et il le fera en appliquant sans réserves les traités et conventions.

Pour répondre aux attentes des victimes et des organisations chargées de protéger leurs droits, des réformes législatives courageuses sont indispensables, en particulier, l'adoption d'une loi spécifique pour l'avortement médicalisée. Certes, la prégnance des facteurs socioculturels constitue un défi important, mais une loi sur l'avortement médicalisée contribuera à respecter les droits humains et de santé des femmes, ainsi qu'à réduire les conséquences sociales, juridiques et sanitaires des grossesses non désirées et des avortements à risque pour les victimes, leurs familles et la société en général. 


\section{Recommandations}

Pour la Taskforce et les acteurs impliqués dans le plaidoyer

- Partager les résultats de l'étude avec les plus hautes autorités de l'Etat (Président) et auprès des personnes influentes (ex : parlementaires).

- Faciliter l'appropriation des données probantes de l'étude par les acteurs institutionnels et de la société civile afin de renforcer le plaidoyer et informer la conception et la mise en œuvre de solutions efficaces pour l'accès à l'avortement médicalisé.

- Réfléchir sur les mécanismes à mettre en place pour valoriser le plus possible la voix des personnes concernées et leurs perceptions de la loi.

- Intégrer les stratégies de prévention dans les activités de chaque acteur en se basant sur les résultats de l'étude.

- Elaborer des supports supplémentaires de sensibilisation avec les informations tirées de l'étude.

- Renforcer le partenariat avec le Ministère de la Justice pour l'élaboration/révision des textes juridiques relatifs à l'avortement médicalisé.

- Renforcer le partenariat avec le Ministère de la famille pour améliorer le contenu des CEDAF au niveau des régions avec:

- Mise en place de psychologues et sociologues.

- Possibilité de logement et de livraison de 1ers secours.

- Mise en place de boutiques de droit, qui pourront également être utilisées pour diffuser l'information sur la loi.

- Renforcer le partenariat avec le Ministère de l'Education pour la sensibilisation dans les écoles:

- Des victimes potentielles sur ce qu'il faut faire en cas de violence et les garçons aux questions de viol.

- Des infirmeries scolaires pour la détection et l'orientation des cas de violence.

- Sensibiliser les populations sur:

- Le caractère délictuel du viol.

- L'accompagnement nécessaire des victimes et encourager le dépôt des plaintes contre les agresseurs.

- Pour les autorités et décideurs

- Harmoniser, au niveau national, les textes relatifs à l'avortement

- Assurer la conformité de la législation sénégalaise en matière d'avortement avec les engagements pris au niveau international.

- Mettre en place des mesures nécessaires pour l'application sévère des peines aux agresseurs.

- Pour les acteurs de mise en œuvre de programmes

- Mettre en place/redynamiser la prise en charge médicale et psycho-sociale des victimes (matérielle et financière). 
- Pour les partenaires techniques et financiers

- Faciliter l'appropriation des données probantes de l'étude par les acteurs institutionnels et de la société civile afin de renforcer le plaidoyer et informer la conception et la mise en œuvre de solutions efficaces pour l'accès à l'avortement médicalisé.

- Etendre l'étude à toutes les régions.

- Financer le plaidoyer et la mise en œuvre d'activités pour l'accès à l'avortement médicalisé.

\section{Limites de l'étude}

Les critères d'éligibilité (notamment âge et site) jugés un peu trop restrictifs. De nombreuses cibles potentielles ne remplissaient pas toujours les critères établis. Par exemple pour le groupe 1, soit les femmes étaient trop jeunes (moins de 18 ans), soit leurs enfants avaient moins d'un an. De même, le fait d'avoir circonscrit l'étude à la région de Dakar a fait que plusieurs cibles potentielles n'ont pas pu être enrôlées.

Cette étude ne se prévaut d'aucune prétention d'exhaustivité ou de généralisation des observations et conclusions qui y sont développées. Néanmoins, les résultats présentés indiquent des tendances non négligeables.

Par ailleurs, certaines cibles n'ont pas pu être identifiées durant la période de la collecte, notamment : les victimes emprisonnées pour avortement illégal et les familles de filles décédées de conséquences sanitaires dramatiques. De ce fait, leur expérience n'a pas pu être documentée. 


\section{Références}

Agence Nationale de la Statistique et de la Démographie (ANSD) et ICF International. 2012. Enquête Démographique et de Santé à Indicateurs Multiples au Sénégal (EDS-MICS) 2010-2011. Calverton, Maryland, USA: ANSD et ICF International.

- Association des Juristes Sénégalaises. Communication sur les ravages de l'interdiction de l'interruption volontaire de grossesse (IVG) au Sénégal.

- FIDH/RADDHO/LSDH, 2014. Sénégal. " Je en veux pas cet enfant, moi je veux aller à l'école ». La prohibition de l'interruption volontaire de grossesse au Sénégal. Décembre 2014.

- Institut Guttmacher, Centre de Recherche pour le Développement Humain (CRDH). L'incidence et la morbidité de l'avortement provoqué au Sénégal. 2015.

- L. JOINET, Question de l'impunité des auteurs des violations des droits de l'homme (civils et politiques), Rapport final pour la Commission des droits de l'homme des Nations Unies, rapport final révisé établi suite à la décision 1996/119 de la Sous-commission, E/CN4/sub2/1997/20/Rev. 1.).

- L. Ndiaye, « Avortement clandestin et cultures négro-africaines. La femme sénégalaise face à la pratique abortive », Ethiopiques-Revue négro-africaine de littérature, philosophie et art, n87, 2011.Rapport semestriel 2013 de la Direction de l'administration pénitentiaire.

Ministère de la Santé et de la Prévention. Division de la Santé de la Reproduction. Rapport de l'évaluation stratégique sur les grossesses non désirées et les avortements à risque au Sénégal, Mai 2010. 
Annexe : Liste des structures membres de la Taskforce

Association des Juristes Sénégalaises (AJS)

Association des Femmes Médecins du Sénégal (AFEMS)

Association Sénégalaise pour le Bien-Etre Familial (ASBEF)

Association Des Infirmiers du Sénégal

Association des Journalistes contre les Viols et Abus (AJVA)

Association des sages-femmes du Sénégal

Association des Journalistes en Santé, Population et Développement

Comité de Lutte contre les Violences faites aux Femmes (CLVF)

Direction de la Santé de la Reproduction et de la Survie de l'Enfant (DSRSE)

Fédération des Associations Féminines du Sénégal (FAFS)

Groupe Pour l'Education et l'Enseignement de la Population (GEEP)

Réseau Islam et Population (RIP)

Réseau Siggil Jigeen (RSJ)

Marie Stoppes International

Population Council

Rencontre Africaine pour la Défense des Droits de l'Homme (RADDHO)

Wildaf

\footnotetext{
POPULATION COUNCIL

Ideas. Evidence. Impact.
} 